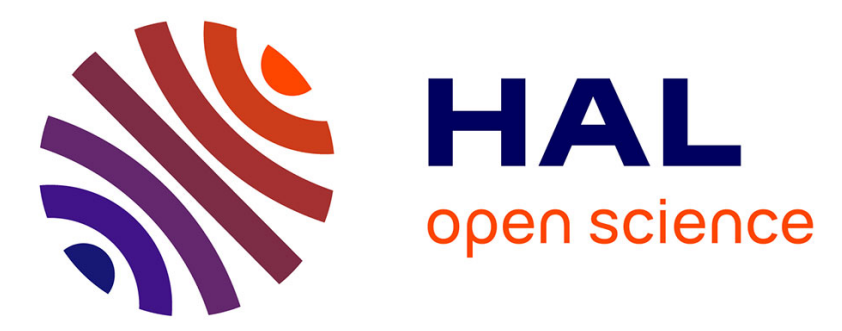

\title{
Effect of haptic supplementation on postural stabilization: A comparison of fixed and mobile support conditions
}

\author{
Inke Marie Albertsen, Jean Jacques Temprado, Eric Berton
}

\section{- To cite this version:}

Inke Marie Albertsen, Jean Jacques Temprado, Eric Berton. Effect of haptic supplementation on postural stabilization: A comparison of fixed and mobile support conditions. Human Movement Science, 2010, 29 (6), pp.999. 10.1016/j.humov.2010.07.013 . hal-00692328

\section{HAL Id: hal-00692328 \\ https://hal.science/hal-00692328}

Submitted on 30 Apr 2012

HAL is a multi-disciplinary open access archive for the deposit and dissemination of scientific research documents, whether they are published or not. The documents may come from teaching and research institutions in France or abroad, or from public or private research centers.
L'archive ouverte pluridisciplinaire HAL, est destinée au dépôt et à la diffusion de documents scientifiques de niveau recherche, publiés ou non, émanant des établissements d'enseignement et de recherche français ou étrangers, des laboratoires publics ou privés. 


\section{Accepted Manuscript}

Effect of haptic supplementation on postural stabilization: A comparison of fixed and mobile support conditions

Inke Marie Albertsen, Jean Jacques Temprado, Eric Berton

PII:

S0167-9457(10)00113-2

DOI:

10.1016/j.humov.2010.07.013

Reference:

HUMOV 1275

To appear in:

Human Movement Science

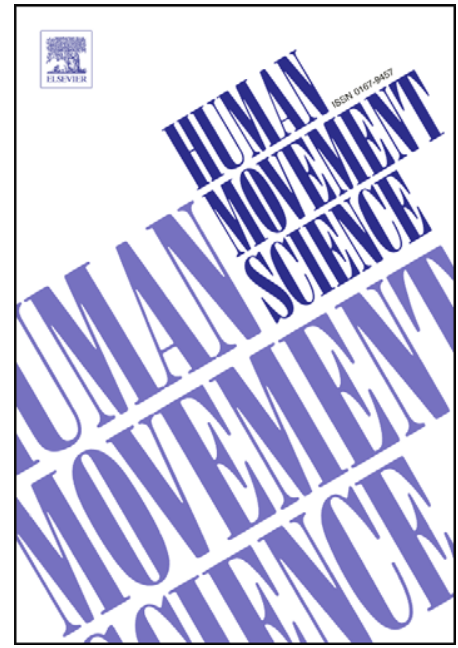

Please cite this article as: Albertsen, I.M., Temprado, J.J., Berton, E., Effect of haptic supplementation on postural stabilization: A comparison of fixed and mobile support conditions, Human Movement Science (2010), doi: 10.1016/ j.humov.2010.07.013

This is a PDF file of an unedited manuscript that has been accepted for publication. As a service to our customers we are providing this early version of the manuscript. The manuscript will undergo copyediting, typesetting, and review of the resulting proof before it is published in its final form. Please note that during the production process errors may be discovered which could affect the content, and all legal disclaimers that apply to the journal pertain. 
Effect of haptic supplementation on postural stabilization: A comparison of fixed and mobile support conditions

Inke Marie Albertsen, Jean Jacques Temprado \& Eric Berton

UMR 6233 «Institut des Sciences du Mouvement » CNRS et Université de la Méditerranée Marseille- France

Running head: Haptic supplementation

Key words: postural stability, haptic information, stick, light grip.

Author correspondence: Jean Jacques Temprado, UMR 6233 "Institut des Sciences du Mouvement", CNRS et Université de la Méditerranée. Faculté des Sciences du Sport, 163 Avenue de Luminy, 13288 Marseille cedex, France. E-mail: jeanjacques.temprado@univmed.fr 


\begin{abstract}
It is well known in the literature of haptic supplementation that a "light touch" (LT) with the index finger on a stable surface increases postural stability. In view of potential application in the domain of mobility aids, it should however be demonstrated that haptic supplementation is effective even when provided by an unstable stick support. The present study aimed to explore the stabilizing effect of a three-digit "light grip" (LG) of different supports (fixed or mobile stick) in young people. Eleven participants $(\mathrm{M}=25.9$ years $)$ were tested in an upright standing task in six experimental conditions in which the mobility of the given support and its resistance in opposite direction to the body movement were manipulated. The RMS variability and the range of postural oscillations were measured. The results confirmed that the stabilizing effect of haptic supplementation is independent from the nature of the support (fixed or mobile) when sufficiently large sway-related contact forces on the fingers are provided. Future applications of this "mobile stick paradigm" to complex situations while targeting different groups of participants may help to approach everyday life situations in which an informational stick could potentially be of assistance to gain stability and mobility.
\end{abstract}




\section{Introduction}

Human bipedal upright stance has been modeled predominantly as a single-joint pendulum rotating around the ankle. Accordingly, upright posture is inherently unstable since the high positioned body mass has to be kept over a relatively small base of support through the continuous exertion of forces on the ground (Balasubramaniam \& Wing, 2002; Horak, 2006; Nashner \& McCollum, 1985). Thus, spatial features of the center of pressure (COP) trajectories, which summarize the behavioral output resulting from the transient forces applied under the feet, are commonly used to capture body oscillations (Horak \& Nashner, 1986).

It is generally accepted that stable upright posture reflects a complex mixture of biomechanical constraints and neural control mechanisms (Horak, 2006). In particular, integration of visual, vestibular, and proprioceptive feedback allows the central nervous system to control postural stability by providing information about the spatial orientation of the body relative to the boundaries of the base of support or to the environment (Peterka, 2002). Consequently, supplementation of any of these systems facilitates the control of body oscillations during static and dynamic upright stance and may be especially important to prevent falls and their dramatic individual consequences on the quality of life of elderly people or populations suffering from neural alterations which affect balance control. With this future objective in mind, the present preliminarily study investigated the benefit of haptic supplementation provided by the light grip of a stick on postural stability of healthy young people.

The term "haptic" sense, introduced by Jeka and Lackner (1995) in the context of postural control, refers to the perceptual sense which combines cutaneous and kinaesthetic inputs from mechanoreceptors embedded in skin, muscles, and joints of the arm and finger while touching or manipulating an object. In their seminal works, Jeka and Lackner (1994, 
1995) demonstrated the role of supplementary haptic information provided by a light touch of the index fingertip on a stable support surface in the control of upright posture. The light touch (LT) paradigm consisted in an active touch $(<1 \mathrm{~N})$ of the index finger on a stationary surface. Specifically, results showed that haptic supplementation reduced the magnitude of COP displacements even though contact forces on the fingertip were too small to mechanically stabilize posture. Afterward, several studies have confirmed the benefit of haptic cues as a means to decrease postural sway (Baccini, Rinaldi, Federighi, Vannucchi, Paci, \& Masotti, 2007; Dickstein, Shupert, \& Horak, 2001; Krishnamoorthy, Slijper, \& Latash, 2002; Rabin, DiZio, Ventura, \& Lackner, 2008).

Theoretical interpretations of the benefits of haptic supplementation have been proposed. For instance, Jeka and Lackner $(1994,1995)$ suggested that an external fingertip contact point on a stable support surface provided to the participants a precise reference frame to detect their own position and to control the spatial orientation of their body. Haptic cues related to body oscillations were effective in this respect since they facilitated the detection of self-motion and body position in the environment and, finally, permitted adaptive corrections leading to a reduction of the magnitude and variability of postural oscillations. Another interpretation was that the central nervous system (CNS) used transient forces at the point of contact with an external fixed support to stabilize posture. Specifically, it has been shown that an LT generates both sway-related changes in contact forces on the fingertip and proprioceptive information regarding arm and finger position, allowing the CNS to anticipate activation of postural muscles and by this means to reduce body oscillations (Dickstein et al., 2001; Jeka \& Lackner 1994; Krishnamoorthy et al., 2002; Lackner, Rabin, \& DiZio, 2001; Rabin et al., 2008). The existence of this feed-forward mechanism has been supported by several works, which showed a constant time lag of $\sim 250-300 \mathrm{~ms}$ between the fingertip force and postural corrections observed by means of COP displacements (Jeka \& Lackner, 1994, 
1995; Lackner et al., 2001). Rabin et al. (2008) showed that, in order to be effective, haptic cues upcoming from transient fingertip contact forces should be completed by congruent arm proprioception. Indeed, they observed that the stabilizing effect of the LT was not affected by restriction of the arm movements, even if the precision contact of the LT was disrupted. In contrast, when arm proprioception was perturbed by vibration of the biceps muscle during the LT, a smaller stabilizing effect was observed. The authors concluded that incongruent information arising from mechanoreceptors of the arm joints and muscles resulted in a biased representation of the body position and thereby in greater postural instability. Overall, the results observed for the LT supplementation procedure suggested that the CNS uses the transient changes of forces arising from the contact of a part of the body with a stationary support surface to detect body oscillations and increase postural stability.

In view of further potential application in the domain of mobility aids, it should however be demonstrated that haptic supplementation is also effective when provided by a cane or a long stick that is, when the support surface is unstable. Indeed, although several authors emphasized the importance of a hand-held cane to provide haptic supplementation and functional spatial information, the question remains whether and in which conditions the CNS can detect the relationship between the environmental surroundings and the oscillating body by the help of a mobile stick, presumably mediating the haptic sensory cues.

To our knowledge, few works have systematically explored the benefit of haptic cues on postural stability by the use of a specifically dedicated "mobile stick" experimental paradigm (see Jeka, Easton, Bentzen, and Lackner (1996) for a noticeable exception). However, the results observed in several studies might lead to expect that an LT on an unstable support could provide useful spatial information to control body oscillations (Boonsinsukh, Panichareon, \& Phansuwan-Pujito, 2009; Jeka et al., 1996; Jeka, 1997; Krishnamoorthy et al., 2002; Lackner et al., 2001). Krishnamoorthy et al. (2002) observed a 
stabilizing effect of a mobile support constituted by a hand-held handle linked via a pulley system to a $3 \mathrm{~kg}$ weight. In this situation, handle displacements and transient horizontal forces arising at the level of the handle were sway-related and helped decreasing body sway. The authors observed that a maximum gain of postural stabilization could be exclusively obtained by the use of a stable LT support. However, even in the absence of a fixed reference point, i.e., without precise information about the position of the unstable support, sway-related transient contact forces based on tissue deformation can be large enough to solely help orientating the body and decreasing sway. Krishnamoorthy et al. (2002) explained their results by the existence of different mechanoreceptors in the skin, which provide sensory cues during touch to inform continuously, on the one hand, about the position of the support (slowly adapting receptors) and, on the other hand, about the direction, amplitude, and velocity of the body oscillations based on tissue deformation (slowly and fast-adapting receptors in combination). A similar conclusion, underlining the importance of sway-related information, can be drawn from the results observed by Reginella, Redfern, and Furman (1999), which showed that erroneous information provided by an oscillating sway-referenced LT support had a destabilizing influence on posture.

These findings suggested that the use of an unstable support such as a mobile stick might provide functional haptic information to stabilize posture when sufficient sway-related transient forces are present. To our knowledge, this hypothesis has never been tested systematically in a "mobile stick" experimental paradigm. However, two studies need to be pointed at, that support the above mentioned hypothesis and that approach certain aspects of such a paradigm. Causing an occasional disruption between the point of contact and the support, Rabin et al. (2008) observed small amplitude movements of the finger on a stationary support surface by fixing the entire arm during LT. Results showed that, even though the finger slipped relative to the stable surface (at force levels less than $3 \mathrm{~N}$ ) a stabilizing effect 
on posture was still observed. Thus, fingertip movements did not preclude sway-related information from being detected and used for spatial orientation of the body. One could point out, that the functional sensory information was gained within a certain stable limited spatial area, within which the COP displacement was kept. Lackner et al. (2001) introduced the notion of a "regional spatial referent", which illustrates this hypothesis in the context of postural stabilization resulting from LT on flexible filaments. They furnished the circular extremity of vertically mounted flexible filaments as a non-rigid LT support, i.e., a slightly deformable and therefore moving support. Even though the stabilizing effect resulting from LT on flexible filaments was less effective than LT on a rigid surface, the authors observed a significant increase in postural stability in both situations. Taken together, the abovementioned findings suggested that a stable reference support is not necessary to improve postural stability when functional transient contact forces are provided. Thus, it encouraged us to study the stabilizing effect of a mobile stick support in a more detailed way.

Jeka et al. (1996) were the first to investigate the possible benefit of a cane as a source of sensory information to improve postural stability. In their experiment, subjects stood in a Romberg tandem stance position and were instructed to lightly grip the handle of a cane $(<2$ $\mathrm{N})$. Two orientation conditions - vertical and slanted in ML direction $\left(70^{\circ}\right.$ with respect to the horizontal) - of a mobile cane, pivoting around its fixed extremity, were assessed. Results showed that the slanted condition was more effective than the vertical condition in reducing postural sway. To explain these results, the authors suggested that, contrary to the vertical cane, the slanted stick did not move in the direction of the participant's body oscillations. Subsequently, it led to functional sway-related contact forces as the result of the resistance offered by the inclined cane to medio-lateral oscillations. This conclusion is consistent with other results showing that stabilization resulting from LT was most effective when force 
changes were generated in the plane of greater instability (Rabin, Bortolami, DiZio, \& Lackner, 1999).

However, a limitation of Jeka et al.'s (1996) study was that the slanted cane was fixed on the ground so that both the handle and the extremity of the stick appeared to be stationary and could consequently not be considered as a mobile support. Thus, potentially functional degrees of freedom were frozen. Moreover, no information was given by Jeka et al. (1996) about the effect of the slanted cane in the antero-posterior direction, in which the handle was actually free to move and, consequently, unstable.

Aims and hypotheses of the experiment

Overall, the above reviewed studies suggested that two types of sensory feedback are entailed in the control process leading to postural stabilization, as a result of light touch contact of fingertip on a support surface (Krishnamoorthy et al., 2002). One is related to the provision of a fixed reference point in space giving rise to an accurate representation of the spatial orientation of the body (e.g., Holden, Ventura, \& Lackner, 1994; Jeka \& Lackner, 1994, 1995; Reginella et al., 1999). The other is involved in the information provided by transient forces developed between the body part and the contact surface that help estimate direction, amplitude, and velocity of the body displacements and, consequently, control postural oscillations (e.g., Krishnamoorthy et al., 2002).

Since a fixed reference point in space could not be provided by a mobile support, we predicted that postural stabilization should even be observed in mobile stick conditions only if contact forces resulting from a resistance in opposite direction to body oscillations are large enough to provide detectable feedback information to the participants related to their body motion. Accordingly, inspired by Jeka et al.'s (1996) "slanted cane paradigm", the present study aimed to test this hypothesis by investigating how haptic supplementation obtained through a three-digit light grip (LG) of a fixed or mobile stick support influenced postural 
stability. By systematically controlling body-related movements of both stick handle (in both the antero-posterior and mediolateral directions) and extremity (in the antero-posterior direction), we presumably manipulated the resistance offered by the stick in a direction opposite to that of the body oscillations. We predicted that postural stabilization should be observed in conditions where transient contact forces were large enough to inform participants about their body oscillations, independent of the nature of the support (i.e., mobile or fixed stick). Moreover, to determine whether the effects of sensory supplementation were sufficiently robust to emerge in this task context, participants were confronted to different conditions in which they were instructed to lightly grip the stick in a natural situation with the feet side-by-side and the eyes open.

\section{Methods}

\section{Participants}

Eleven young participants, four males and seven females, mean age 25.9 years $( \pm 1.9$ $S D$ ) took voluntarily part in the experiment. They were physically active and had no selfdeclared musculoskeletal injuries, or perceptive, cognitive, and motor disorders that might have affected their ability to maintain balance or to understand task instructions. The experimental protocol was presented to all participants, which provided a written consent before undergoing the experiment. The protocol was approved by a local ethics committee and was found to be in accordance with the ethical standards laid down in the declaration of

\section{Helsinki.}

\section{Task and experimental design}

The participants stood on a force platform, eyes open, with both arms fixed by a belt along each side of their body. Their feet were placed at hip-width, side-by-side. The toeholds were positioned at a distance of $20 \mathrm{~cm}$ and an angle of $30^{\circ}$. Adhesive tape was used to mark 
the position on the feet on the platform so that the same task configuration was repeated each trial. Participants were asked to fixate at a point placed at eye height on the wall before them at a distance of $1.50 \mathrm{~m}$. In doing so, they were instructed to adopt a natural upright standing position, to maintain this position as stable as possible, and to focus their attention on this task. For all conditions (but QS), the participants' attention was neither focused on the grip of the stick nor on the extremity of the stick. Prior to each condition participants had a period of familiarization with the task. During this period, participants learned to conform to grip instructions that is, to apply a force underneath a fixed threshold.

Six experimental conditions were run in a randomized order across participants:

(1) A quiet stance condition was used as a reference condition (QS) (Fig. 1A).

(2) A stable support condition (LG), in which participants were instructed to grip the stable stick support lightly with three fingers (index, thumb, and middle finger) of their right hand (Fig. 1B), without exceeding a fixed force threshold (1.6 N). The inclined stick was attached at its rear extremity on an adjustable metal structure so that its height was attuned individually in order to enable participants to grip the handle while keeping their arm straight along the body. This condition presumably furnished sensory cues in both AP and ML direction. However, since, the LG finger configuration differed from the classical index fingertip LT, we had verified in a preliminary control experiment that the LT and the LG on a stable support resulted in equivalent postural stabilization. The mobility of the stick and its resistance to body oscillations in antero-posterior direction was manipulated in four mobile support conditions:

(3) An upright standing task in which participants held the stick lightly on the handle in a roughly horizontal equilibrium position without touching the ground (NTC) (Fig. 1C). Presumably, this condition helped to test the influence of transient inertial forces created by 
the hand-held weight of the stick without any contact to the ground, i.e., in absence of haptic sway-related information by a resistance on the ground in any direction.

(4) A condition of light grip in which the stick handle was mobile while the end of the inclined stick was immobilized on the ground in AP direction (CB) (Fig. 1E). This condition roughly corresponded to the slanted stick condition tested by Jeka et al. (1996). It presumably provided detectable sway-related changes in contact forces on the fingertips in AP direction but not in ML direction.

(5) A light grip condition in which the inclined stick was free to move on either a slippery (CLL) or (6) a rough surface on the ground (CLR) (Fig. 1D). Presumably, in these conditions, the stick extremity was scratching on either a rough (CLR) or a slippery (CLL) surface as the result of body oscillations in the AP direction. These conditions presumably provided more or less easily detectable changes in contact forces in the AP direction but no sway related haptic information in the ML direction. We hypothesized that changes in contact forces, which would result from the movement of the tip of a stick on a limited region on the ground, could provide (depending on the texture of the surface) a sufficient resistance to body sway. This resistance could consequently give rise to functional sensory supplementation and, hence, to postural stabilization (Lackner et al., 2001).

Participants performed three trials of $30 \mathrm{~s}$ in each condition and were given a 30-s rest period between each trial and a 1-min break between each condition. When they were able to stand quietly without exceeding the force threshold the experiment started. The total experimental session lasted about 1 hour.

Insert Fig. 1 about here

Apparatus and measures 
The participant stood on a force platform (AMTI, Advanced Mechanical Technology, Inc., MA, USA) that measured center of pressure (COP) trajectories based on the $x, y$, and $z$ coordinates of the vertical force components and moments generated by the feet at a sampling rate of $200 \mathrm{~Hz}$. The handle of the stick (weight: $400 \mathrm{~g}$, length: $165 \mathrm{~cm}$ ) was instrumented with three micro switches covered by a steel badge $(2.5 \mathrm{~cm})$. A switch dedicated to the index was placed on top of the stick handle (53 cm away from rear extremity) and the two others, dedicated to the thumb and the middle finger, on both lateral sides $(5.5 \mathrm{~cm}$ further towards rear extremity). Each of those switches released when the force exerted by the corresponding finger exceeded $1.6 \mathrm{~N}$ thereby ensuring that grasping forces did not give rise to a mechanical aid. The micro switches were connected to an LED powered by a battery signalling that participant's grip exceeded the fixed threshold (release of, at least, one up to three switches). When the light flashed up during a trial, it was rejected and repeated. No trial was rejected on the basis of this criterion during the experimental session attesting that none of the fingers applied more than $1.6 \mathrm{~N}$. In a preliminary test, that examined the global amount of force applied by the stick extremity on the ground in case of release of the micro switches, a Nano25 transducer (ATI, Industrial automation, Inc., NC, USA) was used that converts force and torque into analog strain gauge signals. Results confirmed that in case the switches released $(>1.6 \mathrm{~N})$, the force applied by the stick extremity did not exceed 2.0 to $2.5 \mathrm{~N}$, which still corresponded to classically fixed force thresholds during LT (keeping in mind that all such trials would have been rejected). The position and height of the stick were both adjustable while keeping a steady angle of $30^{\circ}$ relative to the ground. The rationale for the choice of surface was that the slippery surface should provide shear forces on the stick extremity which were of less magnitude to inform about body sway than those provided by a rough surface. Accordingly, the difference in surface texture between the slippery (plastic) and the rough support (sandpaper: 120 granulation) corresponded to dynamic frictional 
coefficients of 0.37 and 0.58 , respectively. The arms were maintained straight along the body by an adjustable Velcro®- bandage in all conditions and in a constant distance from the body maintained by two foam pads $(12 \mathrm{~cm} \times 8 \mathrm{~cm} \times 1 \mathrm{~cm})$. Data were collected by means of LabView 7.5 (National Instruments ${ }^{\circledR}$, Austin, TX, USA) on a PC and analyzed offline with the help of Matlab 7.0 (The MathWork®, Inc., Natrick, MA, USA).

Center of pressure (COP) trajectories were computed in the antero-posterior $(\mathrm{AP})$ and medio-lateral (ML) directions. From the measured COP trajectories, three dependent variables were calculated for each trial: (1) the root mean square (RMS) in AP and ML direction, which represented the RMS variability and was calculated for each trial after subtracting the average position of COP from each data point, (2) the range of amplitude of the COP displacement calculated by subtracting the greatest from the lowest value of the COP in AP and ML direction, and (3) the mean COP velocity calculated by dividing the total length of the COP trajectory (approximation by summing the distances between two successive points with its $x$ $y$ coordinates linked by a straight line) by the sampling time.

The data obtained for each trial in each condition were averaged for each participant and used to carry out a 6-conditions repeated-measure ANOVA. Moreover, we calculated the percentage decrease of the range in the different conditions that showed a significant stabilizing effect relative to the QS condition. The values obtained for the three conditions (LG, CB, CLR) were transformed in Arcsine values. Newman-Keuls test was used as the post-hoc test. For all analyses, the threshold of significance was set at $p<.05$.

\section{Results}

1) Antero-posterior direction

$R M S$ variability of the COP trajectory 
The analysis revealed an effect of condition, $F(5,50)=6.88, p<.05)$ (Fig. 2). The post-hoc decomposition using the Newman-Keuls test showed that the RMS variability observed in the conditions QS (3.84 mm), NTC (4.02 mm), and CLL (3.55 mm) did not differ significantly $(p>.05)$. In contrast, the RMS variability observed in the conditions QS, NTC, and CLL was higher than in the conditions LG $(2.57 \mathrm{~mm}), \mathrm{CB}(2.29 \mathrm{~mm})$, and CLR $(2.73$ $\mathrm{mm})(p<.05)$. In contrast, the analysis did not reveal any significant difference between the conditions LG, CB, and CLR.

Insert Fig. 2 about here

\section{Range of the COP trajectory}

The analysis revealed an effect of condition, $F(5,50)=9.23, p<.05$. On the one hand, the post-hoc analysis of Newman-Keuls showed that the range did not differ significantly between the conditions QS (18.58 mm), NTC $(19.64 \mathrm{~mm})$, and CLL $(17.73 \mathrm{~mm})(p>.05)$. On the other hand, the range of COP trajectory was higher in these conditions QS, NTC and CLL than in the conditions LG $(13.35 \mathrm{~mm}), \mathrm{CB}(12.77 \mathrm{~mm})$, and CLR $(13.83 \mathrm{~mm})(p<.05)$, which did not differ significantly $(p>.05)$.

2) Medio-lateral direction

RMS variability of the COP trajectory

The analysis revealed no effect of condition, $F(5,50)=1.61, p>.05$. Even

though above the chosen level of significance $(p=.08)$ the RMS variability in the condition LG $(1.7 \mathrm{~mm})$ ended to be smaller than in the condition QS $(2.6 \mathrm{~mm})$.

\section{Range of the COP trajectory}

The analysis revealed an effect of condition, $F(5,50)=2.60, p<.05$. Post-hoc decomposition using the Newman-Keuls test showed that the range observed in the condition 
QS $(13.77 \mathrm{~mm})$ was larger than in the condition LG $(9.57 \mathrm{~mm})(p<.05)$. The conditions QS, NTC, CB, CLL, CLR did not differ significantly $(p>.05)$.

Mean velocity of the COP displacement

The analysis revealed no effect of condition for this variable in any of the two directions $(p<.05)$. Therefore, this variable will not be mentioned again in the following.

Subsequently, we calculated the percentage decrease of the range during a condition compared to the reference condition QS for each participant. Mean percentages of stabilization obtained as the result of QS-LG, QS-CB, and QS-CLR differences were submitted to an arcsine transformation (Abdi, 1987) and then compared using a 3-condition repeated-measures ANOVA. Only the results concerning the range are presented in the following.

\section{1) Antero-posterior direction}

Percentage decrease of the range of the COP trajectory

The analysis revealed no effect of condition $(F(2,20)=0.66, p>.05)$. The percentage decrease observed in the conditions LG (24\%), CB (30\%), and CLR (25\%) did not differ significantly $(p>.05)$.

\section{2) Medio-lateral direction}

Percentage decrease of the range of the COP trajectory

The analysis revealed no effect of condition $(F(2,20)=2.70, p>.05)$. The percentage decrease observed in the conditions LG (27\%), CB (10\%), and CLR (0\%) did not differ significantly $(p>.05)$.

\section{Discussion}

Effects of light grip (LG) on postural stability 
This experiment aimed to test the effect of different conditions of sensory supplementation provided by an LG of either a fixed or a mobile stick. Our main hypothesis was that, independent of the nature of the support, sensory supplementation leads to postural stabilization given that detectable information about body oscillations is provided.

Before discussing the results observed in this respect, it should be noticed that the results of the preliminary control test (not reported here) concerning the effect of an LG on postural stability were reproduced in this experiment. Specifically, postural stabilization was observed in both the antero-posterior and the medio-lateral direction in the LG condition (fixed support) relative to the QS condition. Lower percentage decreases of the range of amplitude (24\% to $27 \%$ ) were however observed for the LG condition as compared with percentages currently observed in the literature for LT conditions (e.g., > 50\%, Jeka \& Lackner, 1995). An explanation of these discrepancies lies in the possible existence of a ceiling effect in the present experiment. Indeed, in Jeka and Lackner's study (1995) postural oscillations were artificially increased by the reduction of the base of support (i.e., the use of a tandem stance position) and by the suppression of visual information. In contrast, in the present experiment, participants performed a more natural upright standing task with the feet side-by-side and the eyes open. A second explanation, not exclusive to the previous one, lies in the fact that the touching arm was strapped to the body and consequently not orientated in the most unstable plane as in Jeka and Lackner's study (1995). This explanation is supported by Rabin et al.'s (1999) results which showed that this arm orientation led to larger changes in joint angles and fingertip forces with a stronger link between the direction of postural oscillations and the provided sensory supplementation. Finally, since in the present experiment both arms of the participants were strapped to the body, it likely limited compensatory movements while holding the stick. Accordingly, freezing the degrees of freedom of the kinematic chain of the arm (i.e., elbow, shoulder) and thus restricting joint 
movements to the wrist and fingers might have reduced available proprioceptive information.

It could thus be speculated that corrections of postural sway were less effective since less sensory information was available to detect body movements. Anyway, as recently observed by Rabin et al. (2008), even if proprioceptive cues arising from the arm involved in the LT were kept constant by immobilizing the arm of the participant, it appeared that information arising from changes in contact forces on the fingers were sufficient to allow a significant decrease in postural oscillations.

Effects of fixed and mobile grip conditions on postural sway in the antero-posterior direction

In the antero-posterior direction, a decrease of the RMS variability and the range of COP displacements were observed in the three conditions LG, CB, and CLR. These results showed that haptic supplementation was effective in improving postural stability, whereas two other conditions did not significantly stabilize posture (LG, CB, CLR vs. NTC, CLL). As, however, all experimental conditions tested in the present study (except for QS) involved an equivalent supra-postural task of lightly gripping the stick support, differences in postural stability observed across conditions cannot be interpreted as the result of goal-oriented postural organization toward the supra-postural task, in order to better achieve the light grip of the stick (Riley, Wong, Mitra, \& Turvey, 1999).

The present results rather do lend credence to our general hypothesis about the benefit of a haptic supplementation independent of the nature of the support (i.e., fixed or mobile). Indeed, among the stabilizing conditions, one of them was provided by a fixed support (LG) and the two others by a mobile support (CB, CLR). These findings suggested that the three conditions of haptic supplementation shared, at least in part, common characteristics with respect to sensory inputs provided to the participants for the control of body oscillations. This interpretation is in agreement with Krishnamoorthy et al.'s (2002) results suggesting that the availability of a fixed support for the LT may not be necessary to reduce sway, if the 
modulations of contact forces during a LT are large enough. Since in the mobile support conditions the CNS could not use a stable reference point to control body oscillations, it can be hypothesized that the three conditions (i.e., including those with mobile support) provided haptic cues by changing contact forces and proprioception related to body oscillations. Specifically, in the two mobile support conditions, the stick encountered a resistance in the direction opposite to that of the body sway either by blockade $(\mathrm{CB})$ or by scratching on the rough surface (CLR). Since these situations produced a comparable stabilizing effect to the one produced by a fixed support, one can hypothesize that this resistance played a prominent role in the control of postural stability by creating sway-related transient contact forces.

Strikingly, no significant difference was found between the conditions CB and CLR concerning RMS variability and the range of body oscillations. In both conditions the handle was free to move in the medio-lateral direction, whereas a further mobility in the anteroposterior direction was added in the CLR condition. We hypothesized that changes in contact forces, resulting from the movement of the tip of a stick on a limited spatial region on the ground, could provide postural stabilization (Lackner et al., 2001). The present results confirmed this hypothesis, thereby extending Jeka et al.'s (1996) findings about the stabilizing effect of a stick fixed on the ground. One could conclude that the sensory information coming from the movement of the stick was attributable to a "regional spatial referent" and was not biased by the mediation of the stick as compared to a fingertip LT (Lackner et al., 2001). This benefit was of the same magnitude as the one produced by an LG on a stable support fixed in space and was even more noteworthy in young healthy participants in an unperturbed situation. These findings suggest that a stabilizing effect on posture can be gained, even in the absence of a stable spatial referent, under the condition that functional transient contact forces are provided (Krishnamoorthy et al., 2002). As expected on the basis of the results of previous studies (Lackner et al., 2001, Rabin et al., 2008), the effects of force changes still persisted 
even when a relative movement between the finger (here the stick extremity) and a small spatial region of the ground was created. Thus, our experimental set-up approached a natural situation of stick use allowing us to merge different aspects of haptic supplementation by an LT to finally confirm a stabilizing effect of an LG of a mobile stick.

The results observed for the range of postural oscillations in the medio-lateral direction (ML) strongly supported the importance of transient contact forces at the fingertips for postural stabilization. Indeed, in the ML direction, no stabilization was observed for the mobile support conditions (i.e., CB and CLR), presumably since no resistance was offered by the stick in the direction opposite to that of the postural oscillations, due to the mobility of the stick handle (see below). These results also suggested that body oscillations in ML and AP directions were controlled separately. Indeed, in some of the mobile stick conditions (CB, CLR), postural stabilization was observed in the AP direction but not in the ML direction.

The results observed in the NTC and CLL conditions also supported the above mentioned interpretation. Indeed, no stabilization effect was observed in both the NTC and CLL conditions. According to the line of reasoning followed above, this suggests that both situations had certain characteristics in common, namely the lack of additional detectable haptic information about body oscillations. Thus, the question remains whether (1) additional sensory information were really lacking in both the NTC and CLL situations due to the nature of the support, or whether (2) the quiet stance situation was not the appropriate situation to make sway-related information detectable for participants in these conditions.

With respect to the NTC condition, the second hypothesis is supported by Hausbeck, Strong, Tamkei, Leonard, and Ustinova's (2009) findings. Hausbeck et al. (2009) observed no postural stabilizing effect of a so-called horizontal "air cane" (similar to NTC) when vision was untroubled, whereas when troubled, gripping the air cane led to a postural stabilization. These results suggest that a more perturbing postural situation would create detectable 
transient inertial forces that appeared to be too small to be detectable or beneficial in this study. From another point of view, one could suggest that the CNS relies more on information provided by small transient inertial contact forces in more demanding or sensory conflicting situations. However, such a speculative hypothesis deserves further investigation, for instance in the context of mechanically perturbed postural situations or during locomotion.

The absence of postural stabilization observed in the CLL condition is more surprising. Indeed, since the stick moved on a slippery ground surface, we expected to observe a (although smaller) stabilizing effect despite the reduction of sensory information as compared to a rough surface (CLR condition). This prediction corresponded to Jeka and Lackner's (1995) findings about the equivalent stabilizing effect on posture of fingertip LT on a support surface with different frictional properties (i.e., slippery and rough). However, in Jeka and Lackner's (1995) experiment, contrary to the present study, no relative movement between finger and support was observed. Furthermore, similar in spirit are the results of Lackner et al. (2001), which revealed a smaller but significant stabilizing effect of flexible filaments that provided a smaller spatial stability and less resistance to touch compared to rigid filaments. To explain however the missing stabilizing effect of the CLL condition in this experiment, it is equally possible that body oscillations were too small to render the information resulting from the movement of the stick on the slippery surface detectable and thus usable for postural control. According to Riley, Stoffregen, Gorcki, and Turvey (1997) an alternative, though speculative, interpretation would be that participants exploited an exploratory strategy in searching for haptic information by making larger body oscillations which would explain the lack of stabilizing effect of the stick on the slippery surface.

Taken together, the present results lead us to distinguish two groups of experimental conditions, hypothetically differing with respect to the presence of functional supplementary sensory information to control postural oscillations. On the one hand, there are the LG, CB, 
CLR conditions in which a resistance was offered to body oscillations by the either fixed or mobile stick support creating sway-related transient contact forces on the fingers. On the other hand, there are the QS, NTC, and CLL conditions, in which no resistance or an insufficient one was offered to body oscillations, e.g., due to the absence or the mobility of the support. Effects of fixed and mobile grip conditions on postural sway in the medio-lateral direction

Results observed in the ML direction across all but one condition diverged from those observed in the AP direction. As expected, stabilization observed in the ML direction differed significantly for the fixed and mobile support conditions. Indeed, mobile support conditions (CB, CLL, CLR, and NTC) failed to improve postural stability. Conversely, the fixed support condition (LG) led to a significant decrease in the range of postural oscillations. These findings can be explained by the absence of changes in fingertip contact forces in the mobile support conditions as the result of ML oscillations. Only in AP direction functional sensory information was provided by the grip of the mobile stick, whereas in ML direction no resistance in opposite direction of body oscillations was provided.

\section{Conclusion}

In summary, the present experiment addressed the issue of how haptic sensory supplementation provided by finger contact with a fixed or mobile (stick) support influenced postural stability. Although the functional role of haptic supplementation has been demonstrated for a long time by different research groups, the present experiment differed from previous LT studies of the literature in at least four important ways. First of all, we used a natural quiet stance situation, thereby extending the demonstration of the powerful stabilizing effect of sensory supplementation even in very stable upright standing conditions. Second, sensory supplementation was provided by a light grip with three fingers permitting to extend the usefulness of sensory supplementation in more natural stick holding situations. Third, across the different conditions, mobility of the handle and the extremity of the stick 
were manipulated independently in both AP and ML directions, so that a firm resistance of the stick to body oscillations could be provided (or not) in both directions. Such strategy permitted to show that the stabilizing effects of sensory information resulted from fingertip force changes rather than just fingertip contact with the stick. Indeed, no postural stabilization was observed in the ML direction when the stick handle was mobile, that is, when no resistance was opposed to body sway. Fourth, by allowing the extremity of the stick to scratch on either a slippery or a rough support surface, we manipulated the resistance of the stick on the ground and, consequently, sensory information fed-back to the participants through changes of fingertip forces. Our results showed that a given level of resistance opposed to body oscillations by the mobile stick (i.e., dynamic frictional coefficient $>0.37$ ) was seemingly required to allow for postural stabilization.

Thus, beyond the effect of the condition LG, the present results lead to identify two conditions with a light grip configuration of the hand provided by a mobile support $(\mathrm{CB}$, CLR) that stabilized posture independently from the nature of support in the AP direction. More specifically, the condition (CLR) in which both the handle and the extremity of the stick were free to move was identified as equally effective to increase postural stability as a fixed support (LG).

These results constitute an encouraging step towards the investigation of the stabilizing effect of haptic supplementation by the help of a mobile stick in various task conditions. Thus, a continuation of the present experiment will consist in applying this "mobile stick paradigm" to dynamic postural situations while targeting different groups of participants. Studying posturally vulnerable elderly people and fallers, in which medio-lateral sway amplitude was found to be the best predictor of future fall risk (Maki, Holliday, \& Topper, 1994), would be of interest in this respect. In particular, investigating the spontaneous positioning of the stick by the elderly during locomotion would contribute to better 
understand the functional use of a lightly touching cane for postural stabilization in dynamic situations. As the present results suggest that body oscillations in ML and AP directions were controlled separately, it would be furthermore interesting to test the possible combined effect of a stick positioned at $45^{\circ}$ in between ML and AP direction.

Additional investigations using the present "mobile stick paradigm" may help to approach everyday life situations in which an informational stick could potentially be of assistance to gain stability and mobility. Experiments are currently in process to address these issues.

\section{Acknowledgment}

We thank Nils Guéguen, Cédrick Bonnet, and Olivier Oullier for their help during the preparation of the experiments and the manuscript. 


\section{References}

Abdi, H. (1987). Introduction au traitement statistique des données expérimentales [Introduction to the statistical treatment of experimental data]. Grenoble: Presses Universitaires de Grenoble.

Baccini, M., Rinaldi, L. A., Federighi, G., Vannucchi, L., Paci, M., \& Masotti, G. (2007). Effectiveness of fingertip light contact in reducing postural sway in older people. Age \& Ageing, 36, 30-35.

Balasubramaniam, R., \& Wing, A. M. (2002). The dynamics of standing balance. Trends in Cognitive Science, 6, 531-536.

Boonsinsukh, R., Panichareon, L., \& Phansuwan-Pujito, P. (2009). Light touch cue through a cane improves pelvic stability during walking in stroke. Archives of Physical Medicine and Rehabilitation, 90, 919-926.

Dickstein, R., Shupert, C. L., \& Horak, F. B. (2001). Fingertip touch improves postural stability in patients with peripheral neuropathy. Gait \& Posture, 14, 238-247.

Hausbeck, C. J., Strong, M. J., Tamkei, L. S., Leonard, W. A., \& Ustinova, K. I. (2009). The effect of additional hand contact on postural stability perturbed environment. Gait \& Posture, 29, 509-513.

Holden, M., Ventura, J., \& Lackner, J. R. (1994). Stabilization of posture by precision contact of the index finger. Journal of Vestibular Research, 4, 285-301.

Horak, F. B. (2006). Postural orientation and equilibrium: what do we need to know about neural control of balance to prevent falls? Age \& Ageing, 35, 7-11.

Horak, F. B., \& Nashner, L. M. (1986). Central programming of postural movements: Adaptation to altered support surface configurations. Journal of Neurophysiology, 55, 13691381.

Jeka, J. J. (1997). Light touch contact as balance aid. Physical Therapy, 77, 476-487.

Jeka, J. J., \& Lackner, J. R. (1994). Fingertip contact influences human postural control. Experimental Brain Research, 100, 495-502.

Jeka, J. J., \& Lackner, J. R. (1995). The role of haptic cues from rough and slippery surfaces in human postural control. Experimental Brain Research, 103, 267-276.

Jeka, J. J., Easton, R. D., Bentzen, B. L., \& Lackner, J. R. (1996). Haptic cues for orientation and postural control in sighted and blind individuals. Perception \& Psychophysics, 58, 409423.

Krishnamoorthy, V., Slijper, H., \& Latash, M. L. (2002). Effects of different types of light touch on postural sway. Experimental Brain Research, 147, 71-79. 
Lackner, J. R., Rabin, E., \& DiZio, P. (2001). Stabilization of posture by precision touch of the index finger with rigid and flexible filaments. Experimental Brain Research, 139, 454464.

Maki, B. E., Holliday, P. J., \& Topper, A. K. (1994). A prospective study of postural balance and risk of falling in an ambulatory and independent elderly population. Journal of Gerontology, 49, 72-84.

Nashner, L. M., \& McCollum, G. (1985). The organization of human postural movements: A formal basis and experimental synthesis. Behavioral and Brain Sciences, 8, 135-167.

Peterka, R. J. (2002). Sensorimotor integration in human postural control. Journal of Neurophysiology, 88, 1097-1118.

Rabin, E., Bortolami, S. B., DiZio, P., \& Lackner, J. R. (1999). Haptic stabilization of posture: Changes in arm proprioception and cutaneous feedback for different arm orientations. Journal of Neurophysiology, 82, 3541-3549.

Rabin, E., DiZio, P., Ventura, J., \& Lackner, J. R. (2008). Influences of arm proprioception and degrees of freedom on postural control with light touch feedback. Journal of Neurophysiology, 99, 595-604.

Reginella, R. L., Redfern, M. S., \& Furman, J. M. (1999). Postural sway with earth-fixed and body-referenced finger contact in young and older adults. Journal of Vestibular Research, 9, 103-109.

Riley, M. A., Wong, S., Mitra, S., \& Turvey, M. T. (1997). Common effects of touch and vision on postural parameters. Experimental Brain Research, 117, 165-170.

Riley, M. A., Stoffregen,T. A., Grocki, M. J., \& Turvey, M. T. (1999). Postural stabilization for the control of touching. Human Movement Science, 18, 795-817. 


\section{Captions}

Figure 1: Experimental set-up and conditions (see text for details) A) Quiet stance condition. B) $\boldsymbol{L} \boldsymbol{G}$ condition. C) NTC condition. D) CLR and $\boldsymbol{C L L}$ conditions. E) $\boldsymbol{C B}$ condition.

Figure 2: RMS of the COP trajectory in the antero-posterior direction. The conditions are ordered according to their difference with the QS condition. QS: quiet stance, LG: light grip on fixed stick, $\boldsymbol{C B}$ : mobile stick blocked on the tip, CLR: mobile stick on rough surface, CLL: mobile stick on slippery surface and NTC: hand-held horizontal stick.

Figure 3: RMS of the COP trajectory in medio-lateral direction. The conditions are ordered according to their difference with the QS condition. QS: quiet stance, LG: light grip on fixed stick, NTC: hand-held horizontal stick, CB: mobile stick blocked on the tip, CLL: mobile stick on slippery surface and $\boldsymbol{C L R}$ : mobile stick on rough surface. 
Figure1
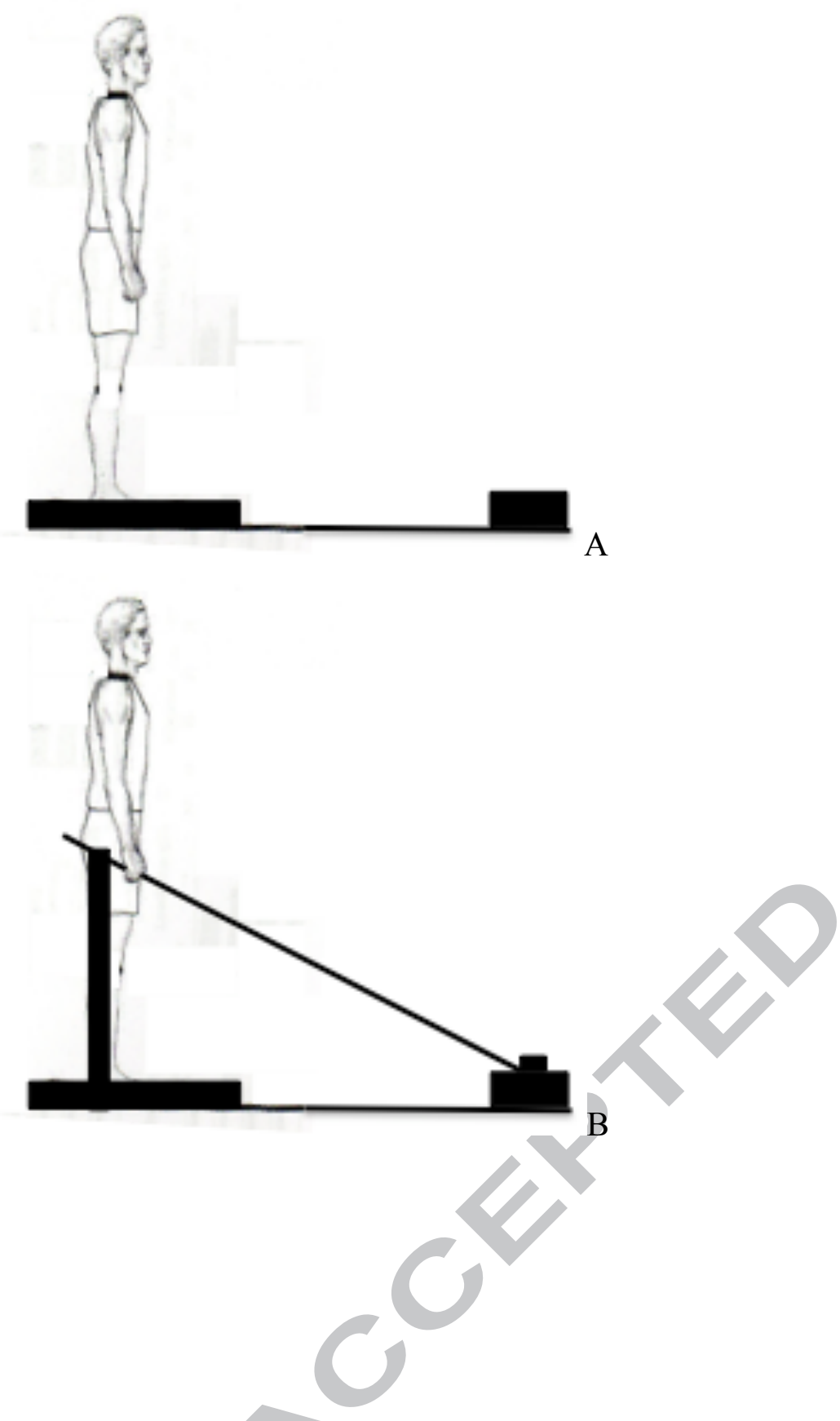

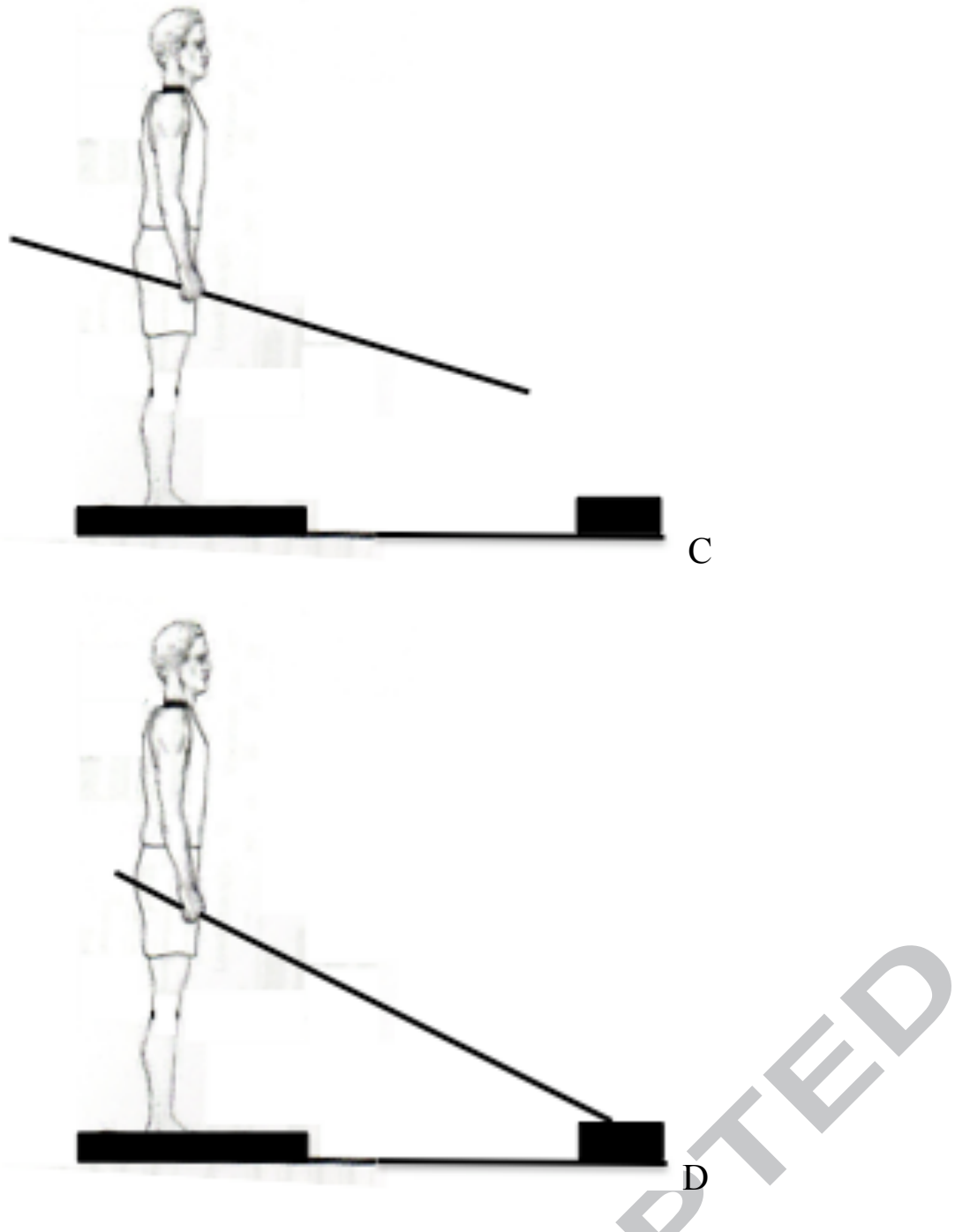
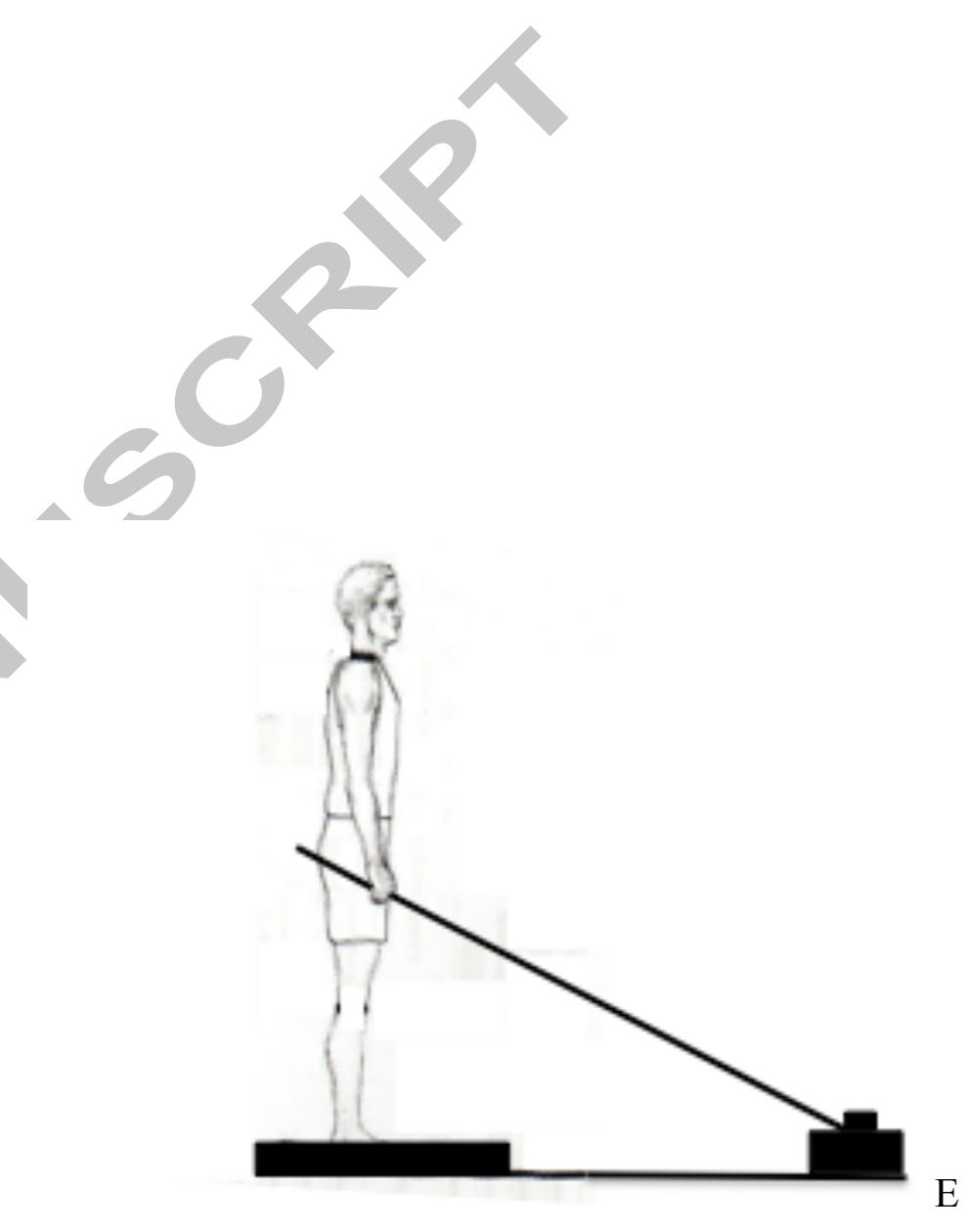
Figure2

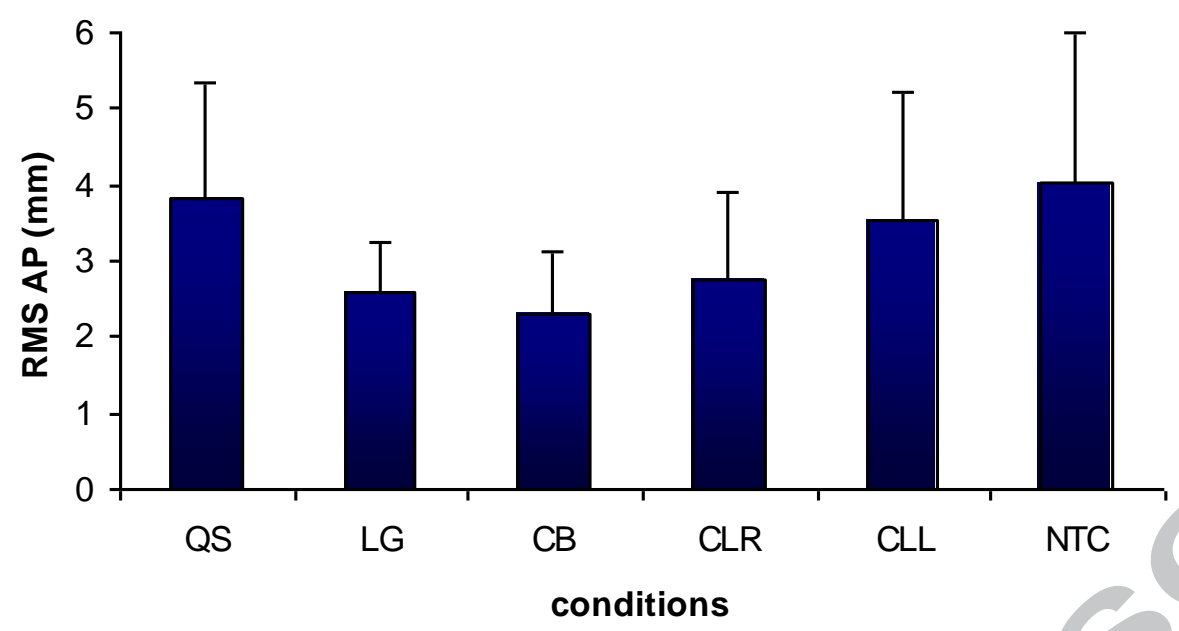

